\title{
ON PARTIALLY ORDERED GROUPS SATISFYING THE RIESZ INTERPOLATION PROPERTY ${ }^{1}$
}

\section{J. ROGER TELLER}

I. Preliminaries. Throughout this paper po-group will mean partially ordered abelian group. A po-group $G$ is semi-closed if $g \in G$ and $n g \geqq 0$ for some $n>0$ implies $g \geqq 0$. $G$ is directed if, whenever $g_{1}$ and $g_{2}$ are elements of $G$, there is an element $g \in G$ such that $g \geqq g_{1}$ and $g \geqq g_{2}$. A subset $B$ of $G$ is lower directed (upper directed) if, whenever $a, b \in B$, there is an element $x \in B$ such that $x \leqq a$ and $x \leqq b(x \geqq a$ and $x \geqq b$ ). $B$ is a dual ideal of $G$ if $b \in B$ and $a \geqq b$ implies $a \in B$. If $A$ is a convex subgroup of $G$, then a natural order is defined in $G / A$ by setting $X \in G / A$ positive if $X$ contains a positive element of $G$. All quotient structures will be ordered in this manner. For the po-group $G, G^{+}=\{x \in G: x \geqq 0\}$.

A po-set $S$ satisfies the Riesz Interpolation Property if, whenever $x_{1}, \cdots, x_{m}, y_{1}, \cdots, y_{n}$ are elements of $S$ and $x_{i} \leqq y_{j}$ for $1 \leqq i \leqq m$, $1 \leqq j \leqq n$, then there is an element $z \in S$ such that $x_{i} \leqq z \leqq y_{j}$. Birkhoff $[1$, p. 328], lists some conditions that are equivalent to the Interpolation Property. The following lemma includes those conditions given by Birkhoff.

Lemma 1.1. If $G$ is a po-group then the following are equivalent.

(1) Riesz Interpolation Property,

(2) (Decomposition Property). If $a, b, x \in G^{+}$and $0 \leqq x \leqq a+b$, then there exist elements $a^{\prime}, b^{\prime} \in G$ such that $x=a^{\prime}+b^{\prime}$ and $0 \leqq a^{\prime} \leqq a, 0 \leqq b^{\prime}$ $\leqq b$.

(3) If $I(a)=\{x \in G \mid 0 \leqq x \leqq a\}$, then $I(a)+I(b)=I(a+b)$.

(4) If $U\left(a_{1}, \cdots, a_{n}\right)=\left\{x \in G \mid x \geqq a_{i}, \quad 1 \leqq i \leqq n\right\}, \quad a_{i} \in G$, then $U\left(a_{1}, \cdots, a_{n}\right)$ is lower directed.

(5) $U\left(a_{1}, \cdots, a_{n}\right)+U\left(b_{1}, \cdots, b_{m}\right)=U\left(a_{i}+b_{j}, 1 \leqq i \leqq n, 1 \leqq j \leqq m\right)$.

(6) If $[x, y]=\{z \in G \mid x \leqq z \leqq y\}$ then for $x, y, u, v \in G,[x, y]+[u, v]$ $=[x+u, y+v]$.

(7) If $a_{1}+a_{2}=b_{1}+b_{2}$, where $a_{1}, a_{2}, b_{1}, b_{2} \in G^{+}$, then there are elements $x, y, u, v \in G^{+}$such that 1964.

Presented to the Society, January 26, 1965; received by the editors November 2,

1 This research was supported in part by N.S.F. grants GP 41 and GP 1634 and represents a portion of the author's Ph.D. dissertation. The author wishes to thank Professors P. Conrad and L. Fuchs for their assistance in the preparation of this paper. 


$$
\begin{array}{ll}
a_{1}=x+y, & b_{1}=x+u, \\
a_{2}=u+v, & b_{2}=y+v .
\end{array}
$$

We omit the straightforward proof and note that (1)-(5) are equivalent if $G$ is not abelian.

Definition. A Riesz group is a semi-closed, po-group that satisfies the conditions of Lemma 1.1.

The purpose of this paper is to give some of the elementary properties of Riesz groups. In (5), the extension problem for lattice ordered groups ( $l$-groups) was considered. In $\S I I$ we give necessary and sufficient conditions that a po-extension of one Riesz group by another be a Riesz group. In the final section some nontrivial examples of Riesz groups are given.

It is clear from the convexity property that any convex subgroup of a Riesz group is itself a Riesz group. Hence, it is equally clear that the cardinal sum, $G=A+B$, is a Riesz group if and only if both $A$ and $B$ are Riesz groups.

LEMMA 1.2. If $G$ is a Riesz group and $A$ is a convex, directed subgroup of $G$, then $G / A$ is a Riesz group.

Proof. Let $G$ be a Riesz group and $A$ be a convex, directed subgroup of $G$. Suppose $X=x+A, Y=y+A$ and $Z=z+A$ are positive elements of $G / A$ such that $0 \leqq Z \leqq X+Y$. Then there are elements $a_{i}, i=1,2,3,4$, in $A$ such that $x+a_{1}, y+a_{2}$, and $z+a_{3}$ are positive elements of $G$ and $0 \leqq z+a_{3} \leqq x+y+a_{4}$. Since $A$ is directed there exists $a \in A$ such that $a \geqq 0, a_{1}, a_{2}, a_{4}$. Thus, $0 \leqq z+a_{3} \leqq x+y+a_{4}$ $\leqq(x+a)+(y+a)$ where $0 \leqq x+a_{1} \leqq x+a$ and $0 \leqq y+a_{2} \leqq y+a$. Since $G$ is a Riesz group, there are elements $b_{1}, b_{2} \in G$ such that $b_{1}+b_{2}$ $=z+a_{3}$ and $0 \leqq b_{1} \leqq x+a, 0 \leqq b_{2} \leqq y+a$. By setting $X^{\prime}=b_{1}+A$ and $Y^{\prime}=b_{2}+A$ we have $0 \leqq X^{\prime} \leqq X, \quad 0 \leqq Y^{\prime} \leqq Y$. Moreover, $X^{\prime}+Y^{\prime}$ $=\left(b_{1}+A\right)+\left(b_{2}+A\right)=z+a_{3}+A=Z$. Thus, $G / A$ satisfies (2) of Lemma 1.1.

To show $G / A$ is semi-closed, let $X=x+A \in G / A$ and suppose $n X \geqq 0$ where $n>0$. Then there is an element $a \in A$ such that $n x+a$ $\geqq 0$ in $G$. Let $b \in A$ such that $b \geqq a, 0$. Then $n b \geqq b \geqq a$ so $n(x+b)$ $=n x+n b \geqq n x+a \geqq 0$. Since $G$ is semi-closed this implies $x+b \geqq 0$ and thus $X \geqq 0$ in $G / A$. This completes the proof.

If $G$ is a semi-closed po-group and $D$ is the $d$-closure of $G$, i.e., $D$ is the minimal divisible group that contains $G$, then the order of $G$ can be extended to a semi-closed partial order of $D$ by defining $d \geqq 0$ in $D$ if $n d \geqq 0$ in $G$ for some $n>0$. 
Lemma 1.3. If $G$ is a Riesz group and $D$ is the $d$-closure of $G$, then $D$ is a Riesz group.

This follows by a straightforward application of property (2) of Lemma 1.1.

II. Extensions of Riesz groups. The notation used in this section and in the next may be found in (5).

A po-group $G$ is a partially ordered extension (po-extension) of a po-group $A$ by a po-group $\Delta$ if there is an order preserving homomorphism $\pi$ of $G$ onto $\Delta$ with kernel $A$ such that $\pi$ induces an order preserving isomorphism of $G / A$ with $\Delta$. If $G$ is a po-extension of $A$ by $\Delta$ whose representative function is $r(\alpha)$ and whose derived factor function is $f(\alpha, \beta)$, then we obtain, for each $\alpha \in \Delta^{+}$, the order sets $Q(\alpha)=\{a \in A \mid r(\alpha)+a \geqq 0\}$, which satisfy

(i) $Q(\alpha) \neq \varnothing$,

(ii) $Q(\alpha)+Q(\beta)+f(\alpha, \beta) \subseteq Q(\alpha+\beta)$,

(iii) $Q(\theta)=A^{+}$, where $\theta$ is the identity of $\Delta$.

We denote a po-extension by $G=(A, \Delta, f, Q)$. Note that $Q(\alpha)$ is a dual ideal of $A$.

Definition. A po-extension $G=(A, \Delta, f, Q)$ is a Riesz extension if $G$ is a Riesz group and $A$ is a directed subgroup of $G$.

In the proof of the following theorem we need

Lemma 2.1. If $X$ and $Y$ are dual ideals of a po-group $G$ and $Z$ is a lower directed subset of $G$ then $(X \cap Y)+Z=(X+Z) \cap(Y+Z)$.

The proof is left to the reader.

Theorem 2.1. Let $A$ and $\Delta$ be Riesz groups, $A$ directed, and let $G=(A, \Delta, f, Q)$ be a semi-closed po-extension of $A$ by $\Delta$. Then $G$ is a Riesz extension if and only if, for $\alpha, \beta \in \Delta^{+}$,

(1) $Q(\alpha)+Q(\beta)+f(\alpha, \beta)=Q(\alpha+\beta)$,

(2) $Q(\alpha)$ is lower directed for each $\alpha \in \Delta^{+}$.

Proof. Suppose $G$ is a Riesz extension of $A$ by $\Delta$ and $\alpha, \beta \in \Delta^{+}$. To show (1) we need only show $Q(\alpha)+Q(\beta)+f(\alpha, \beta) \supseteq Q(\alpha+\beta)$ since $G$ is a po-extension. To this end let $x \in Q(\alpha+\beta), u \in Q(\alpha)$ and $v \in Q(\beta)$. Since $A$ is directed there are elements $a, b \in A$ such that $a \geqq u, a \geqq x$, $b \geqq v$, and $b \geqq(a-x)-f(\alpha, \beta)$. Thus, $a \in Q(\alpha)$ and $b \in Q(\beta)$. Moreover, $x \leqq a+b+f(\alpha, \beta)$ so $(0, \theta) \leqq(x, \alpha+\beta) \leqq[a+b+f(\alpha, \beta), \alpha+\beta]=(a, \alpha)$ $+(b, \beta)$. Since $G$ is a Riesz extension there are elements $\left(a^{\prime}, \alpha^{\prime}\right)$ and $\left(b^{\prime}, \beta^{\prime}\right)$ in $G$ such that $(x, \alpha+\beta)=\left(a^{\prime}, \alpha^{\prime}\right)+\left(b^{\prime}, \beta^{\prime}\right)$ and $(0, \theta) \leqq\left(a^{\prime}, \alpha^{\prime}\right)$ $\leqq(a, \alpha),(0, \theta) \leqq\left(b^{\prime}, \beta^{\prime}\right) \leqq(b, \beta)$. Thus, $\theta \leqq \alpha^{\prime} \leqq \alpha, \theta \leqq \beta^{\prime} \leqq \beta$ and since 
$\alpha^{\prime}+\beta^{\prime}=\alpha+\beta$ it follows that $\alpha^{\prime}=\alpha$ and $\beta^{\prime}=\beta$. Consequently, $a^{\prime} \in Q(\alpha)$, $b^{\prime} \in Q(\beta)$ and $x=a^{\prime}+b^{\prime}+f(\alpha, \beta) \in Q(\alpha)+Q(\beta)+f(\alpha, \beta)$ and (1) holds.

To show (2) let $\alpha \in \Delta^{+}$and $a, b \in Q(\alpha)$. Let $x \in A$ such that $x \geqq 0$, and $x \geqq b-a$. Then $(0, \theta) \leqq(a, \alpha) \leqq(x+a, \alpha)=(b, \alpha)+(x-b+a, \theta)$ so there are elements $\left(a^{\prime}, \alpha^{\prime}\right)$ and $\left(b^{\prime}, \beta^{\prime}\right)$ in $G$ such that $(a, \alpha)=\left(a^{\prime}, \alpha^{\prime}\right)$ $+\left(b^{\prime}, \beta^{\prime}\right)$ and $(0, \theta) \leqq\left(a^{\prime}, \alpha^{\prime}\right) \leqq(b, \alpha),(0, \theta) \leqq\left(b^{\prime}, \beta^{\prime}\right) \leqq(x-b+a, \theta)$. Thus, $\beta^{\prime}=\theta$ and $\alpha^{\prime}=\alpha, a^{\prime} \in Q(\alpha)$ and $(b, \alpha) \geqq\left(a^{\prime}, \alpha\right)$. Therefore, $b \geqq a^{\prime}$ and $a \geqq a^{\prime}$ since $(a, \alpha) \geqq\left(a^{\prime}, \alpha\right)$. Hence, $Q(\alpha)$ is lower directed.

Now suppose $G=(A, \Delta, f, Q)$ is a semi-closed po-extension that satisfies (1) and (2). To show $G$ is a Riesz extension we will show $G$ satisfies the Decomposition Property. To this end let $(a, \alpha),(b, \beta)$ and $(c, \gamma)$ be positive elements of $G$ such that $(0, \theta) \leqq(c, \gamma) \leqq(a, \alpha)$ $+(b, \beta)$. Then $\theta \leqq \gamma \leqq \alpha+\beta$ where $\alpha, \beta \in \Delta^{+}$, so there are elements $\alpha^{\prime}, \beta^{\prime} \in \Delta^{+}$such that $\gamma=\alpha^{\prime}+\beta^{\prime}$ and $\theta \leqq \alpha^{\prime} \leqq \alpha, \theta \leqq \beta^{\prime} \leqq \beta$. Thus, $c \in Q\left(\alpha^{\prime}+\beta^{\prime}\right)=Q\left(\alpha^{\prime}\right)+Q\left(\beta^{\prime}\right)+f\left(\alpha^{\prime}, \beta^{\prime}\right)$ so $c=c_{1}+f\left(\alpha^{\prime}, \beta^{\prime}\right)$ where $c_{1} \in Q\left(\alpha^{\prime}\right)+Q\left(\beta^{\prime}\right)$. Also, $a+b+f(\alpha, \beta)-c-f(\gamma,-\gamma)+f(\alpha+\beta,-\gamma)$ $\in Q(\alpha+\beta-\gamma)$ and it follows that $a+b+f(\alpha, \beta)-c \in Q\left[\left(\alpha-\alpha^{\prime}\right)\right.$ $\left.+\left(\beta-\beta^{\prime}\right)\right]+f\left[\left(\alpha-\alpha^{\prime}\right)+\left(\beta-\beta^{\prime}\right), \alpha^{\prime}+\beta^{\prime}\right]$. Let $\alpha^{\prime \prime}=\alpha-\alpha^{\prime}$ and $\beta^{\prime \prime}=\beta$ $-\beta^{\prime}$. Then $a+b+f\left(\alpha^{\prime \prime}+\alpha^{\prime}, \beta^{\prime \prime}+\beta^{\prime}\right)-c \in Q\left(\alpha^{\prime \prime}+\beta^{\prime \prime}\right)+f\left(\alpha^{\prime \prime}+\beta^{\prime \prime}\right.$, $\left.\alpha^{\prime}+\beta^{\prime}\right)=Q\left(\alpha^{\prime \prime}\right)+Q\left(\beta^{\prime \prime}\right)+f\left(\alpha^{\prime \prime}, \beta^{\prime \prime}\right)+f\left(\alpha^{\prime \prime}+\beta^{\prime \prime}, \alpha^{\prime}+\beta^{\prime}\right)$. Thus, $a+b$ $+f\left(\alpha^{\prime \prime}+\alpha^{\prime}, \beta^{\prime \prime}+\beta^{\prime}\right)-c_{1}-f\left(\alpha^{\prime}, \beta^{\prime}\right)-f\left(\alpha^{\prime \prime}, \beta^{\prime \prime}\right)-f\left(\alpha^{\prime \prime}+\beta^{\prime \prime}, \alpha^{\prime}+\beta^{\prime}\right)$ $=a+b-c_{1}-f\left(\alpha^{\prime \prime}, \alpha^{\prime}\right)-f\left(\beta^{\prime \prime}, \beta^{\prime}\right) \in Q\left(\alpha^{\prime \prime}\right)+Q\left(\beta^{\prime \prime}\right)$. Let $a_{1}=a-f\left(\alpha^{\prime \prime}, \alpha^{\prime}\right)$ and $b_{1}=b-f\left(\beta^{\prime \prime}, \beta^{\prime}\right)$. Then we have $a_{1}+b_{1}-c_{1} \in Q\left(\alpha^{\prime \prime}\right)+Q\left(\beta^{\prime \prime}\right)$.

Now $a_{1}+f\left(\alpha^{\prime \prime}, \alpha^{\prime}\right)=a \in Q(\alpha)=Q\left(\alpha^{\prime \prime}\right)+Q\left(\alpha^{\prime}\right)+f\left(\alpha^{\prime \prime}, \alpha^{\prime}\right)$ and $a_{1}$ $\in Q\left(\alpha^{\prime \prime}\right)+Q\left(\beta^{\prime \prime}\right)+c_{1}-b_{1}$ so, by Lemma 2.1,

$$
a_{1} \in Q\left(\alpha^{\prime \prime}\right)+\left\{Q\left(\alpha^{\prime}\right) \cap\left[Q\left(\beta^{\prime \prime}\right)+c_{1}-b_{1}\right]\right\} \text {. }
$$

Thus, $a_{1}=\bar{a}+x$ where $\bar{a} \in Q\left(\alpha^{\prime \prime}\right)$ and $x \in Q\left(\alpha^{\prime}\right) \cap\left[Q\left(\beta^{\prime \prime}\right)+c_{1}-b_{1}\right]$ so it follows that $b_{1} \in Q\left(\beta^{\prime \prime}\right)+c_{1}-x=Q\left(\beta^{\prime \prime}\right)+Q(\theta)+c_{1}-x$. Also, $b_{1}+$ $f\left(\beta^{\prime \prime}, \beta^{\prime}\right)=b \in Q(\beta)=Q\left(\beta^{\prime \prime}\right)+Q\left(\beta^{\prime}\right)+f\left(\beta^{\prime \prime}, \beta^{\prime}\right)$ so by Lemma 2.1,

$$
b_{1} \in Q\left(\beta^{\prime \prime}\right)+\left\{Q\left(\beta^{\prime}\right) \cap\left[Q(\theta)+c_{1}-x\right]\right\} .
$$

Hence, $b_{1}=b+y$ where $b \in Q\left(\beta^{\prime \prime}\right)$ and $y \in Q\left(\beta^{\prime}\right) \cap\left[Q(\theta)+c_{1}-x\right]$. Consequently, $y=p+c_{1}-x$ where $0 \leqq p \in A$, so $c_{1}=y+x-p \in Q\left(\beta^{\prime}\right)+x-p$ $=Q\left(\beta^{\prime}\right)+Q(\theta)+x-p$. Since $c_{1} \in Q\left(\alpha^{\prime}\right)+Q\left(\beta^{\prime}\right)$ it follows from Lemma 2.1 that

$$
c_{1} \in Q\left(\beta^{\prime}\right)+\left\{Q\left(\alpha^{\prime}\right) \cap[Q(\theta)+x-p]\right\}
$$

which implies $c_{1} \in Q\left(\beta^{\prime}\right)+w$ where $w \in Q\left(\alpha^{\prime}\right) \cap[Q(\theta)+x-p]$. Thus, $w=m+x-p$ where $m \geqq 0$ and so $w \geqq x-p$.

We have $w, x \in Q\left(\alpha^{\prime}\right)$ so by (2) there is an element $z \in Q\left(\alpha^{\prime}\right)$ such 
that $z \leqq x$ and $z \leqq w$. Also, $x-p \leqq w$ and clearly, $x-p \leqq x$. By the Riesz Interpolation Property there is an element $a^{\prime} \in A$ such that $x-p \leqq a^{\prime}, z \leqq a^{\prime}, a^{\prime} \leqq w$ and $a^{\prime} \leqq x$.

Now $z \leqq a^{\prime}$ implies $a^{\prime} \in Q\left(\alpha^{\prime}\right)$ and $a^{\prime} \leqq x$ implies $a-a^{\prime} \geqq a-x=a+\bar{a}$ $-a_{1}=\bar{a}+f\left(\alpha^{\prime \prime}, \alpha^{\prime}\right) \in Q\left(\alpha^{\prime \prime}\right)+f\left(\alpha^{\prime \prime}, \alpha^{\prime}\right)$. Since $(a, \alpha)-\left(a^{\prime}, \alpha^{\prime}\right)=\left[a-a^{\prime}\right.$ $\left.-f\left(\alpha^{\prime},-\alpha^{\prime}\right)+f\left(\alpha,-\alpha^{\prime}\right), \alpha-\alpha^{\prime}\right]=\left[a-a^{\prime}-f\left(\alpha^{\prime \prime}, \alpha^{\prime}\right), \alpha^{\prime \prime}\right] \geqq(0, \theta)$ it follows that $(0, \theta) \leqq\left(a^{\prime}, \alpha^{\prime}\right) \leqq(a, \alpha)$. Since $w \geqq a^{\prime}$ it follows, in a similar manner, that $(0, \theta) \leqq\left(a^{\prime}, \alpha^{\prime}\right) \leqq(c, \gamma)$ and hence, $(c, \gamma)-\left(a^{\prime}, \alpha^{\prime}\right)$ $\geqq(0, \theta)$.

Finally, $a^{\prime} \geqq x-p$ so $b_{1}-\left(c_{1}-a^{\prime}\right) \geqq b_{1}-\left[c_{1}-(x-p)\right]=b \in Q\left(\beta^{\prime \prime}\right)$ which implies $b-f\left(\beta^{\prime \prime}, \beta^{\prime}\right)-c_{1}-a^{\prime} \in Q\left(\beta^{\prime \prime}\right)$. Thus,

$$
\begin{aligned}
(b, \beta)- & {\left[(c, \gamma)-\left(a^{\prime}, \alpha^{\prime}\right)\right] } \\
= & (b, \beta)-\left[c-a^{\prime}-f\left(\alpha^{\prime},-\alpha^{\prime}\right)+f\left(\gamma,-\alpha^{\prime}\right), \gamma-\alpha^{\prime}\right] \\
= & \left\{b-c+a^{\prime}+f\left(\alpha^{\prime},-\alpha^{\prime}\right)-f\left(\gamma,-\alpha^{\prime}\right)-f\left[\gamma-\alpha^{\prime},-\left(\gamma-\alpha^{\prime}\right)\right]\right. \\
& \left.\quad+f\left(\beta,-\gamma+\alpha^{\prime}\right), \beta-\gamma+\alpha^{\prime}\right\} \\
= & {\left[b-c+a^{\prime}+f\left(\alpha^{\prime}, \beta^{\prime}\right)-f\left(\beta^{\prime},-\beta^{\prime}\right)+f\left(\beta,-\beta^{\prime}\right), \beta^{\prime \prime}\right] } \\
= & {\left[b-c_{1}+a^{\prime}-f\left(\beta^{\prime \prime}, \beta^{\prime}\right), \beta^{\prime \prime}\right] \geqq(0, \theta) . }
\end{aligned}
$$

Hence, $(b, \beta) \geqq\left[(c, \gamma)-\left(a^{\prime}, \alpha^{\prime}\right)\right]$.

In conclusion we have, $(c, \gamma)=\left(a^{\prime}, \alpha^{\prime}\right)+\left[(c, \gamma)-\left(a^{\prime}, \alpha^{\prime}\right)\right]$ where $(0, \theta) \leqq\left(a^{\prime}, \alpha^{\prime}\right) \leqq(a, \alpha)$ and $(0, \theta) \leqq\left[(c, \gamma)-\left(a^{\prime}, \alpha^{\prime}\right)\right] \leqq(b, \beta)$ as desired. The proof is complete.

The author wishes to thank Professor A. H. Clifford for his help in simplifying the above proof.

III. Examples. It is clear, by (4) of Lemma 1.1 that every $l$-group is a Riesz group. Other examples of Riesz groups can easily be constructed from $l$-groups in the following way.

Let $A$ and $\Delta$ be $l$-groups and $G=A \oplus \Delta$. Define $(a, \alpha)$ positive in $G$ if $\alpha>\theta$ or $\alpha=\theta$ and $a \geqq 0$. Clearly $G$ is semi-closed and $G$ is a poextension of $A$ by $\Delta$ where $Q(\alpha)=A$ if $\alpha>\theta$ and $Q(\theta)=A^{+}$. Thus, by the previous theorem, $G$ is a Riesz group.

It is well known that $G$ is an $l$-group if and only if $\Delta$ is an ordered group.

The remaining examples are all Hahn-type po-groups which are defined as follows. Let $\Gamma$ be a po-set and, for each $\gamma \in \Gamma$, let $R_{\gamma}$ be a nontrivial po-group. Let $V=V\left(\Gamma, R_{\gamma}\right)$ be the following subset of the large direct sum of the $R_{\gamma}$. An element $v=(\cdots, v(\gamma), \cdots) \in V$ if and only if $S(v)=\{\gamma \in \Gamma \mid v(\gamma) \neq 0\}$ contains no infinite ascending sequences. This is the same as saying every nonempty subset of $S(v)$ 
contains at least one maximal element. If $v \in V$ then $v(\gamma), \gamma \in \Gamma$, is a maximal component of $v$ if $v(\gamma) \neq 0$ and $v(\alpha)=0$ for all $\alpha>\gamma$. A nonzero element $v$ is positive if each maximal component of $v$ is positive. In $\left[3\right.$, p. 145] it is shown that $V\left(\Gamma, R_{\gamma}\right)$ is a po-group. If $\alpha, \beta \in \Gamma$ such that $\alpha \geq \beta$ and $\beta \geq \alpha$ then we write $\alpha|| \beta$. If $B$ is a subset of a pogroup $G$ then $D I[B]=\{g \in G \mid g \geqq b$, for some $b \in B\}$.

THEOREM 3.1. If $\Gamma$ is a po-set with a finite number of maximal chains and for each $\gamma \in \Gamma, R_{\gamma}$ is an o-group, then $V=V\left(\Gamma, R_{\gamma}\right)$ is a Riesz group.

Proof. Suppose $\Gamma$ has $n<\infty$ maximal chains and, for each $\gamma \in \Gamma$, $R_{\gamma}$ is an o-group. The proof is by induction on $n$.

If $n=1$, then $\Gamma$ is a chain and $V$ is an o-group by [3, p. 147]. So suppose the theorem is true for all po-sets with fewer than $n$ maximal chains. Let $C$ be a maximal chain of $\Gamma, C^{\prime}=\{\gamma \in C \mid$ there exist $\delta_{1}, \delta_{2} \in \Gamma, \delta_{1}|| \delta_{2}$ and $\left.\gamma>\delta_{1}, \gamma>\delta_{2}\right\}, \Gamma^{\prime}=C \backslash C^{\prime}$ and $\Gamma^{\prime \prime}=\Gamma \backslash \Gamma^{\prime}$. We first show $\Gamma^{\prime \prime}$ has fewer than $n$ maximal chains.
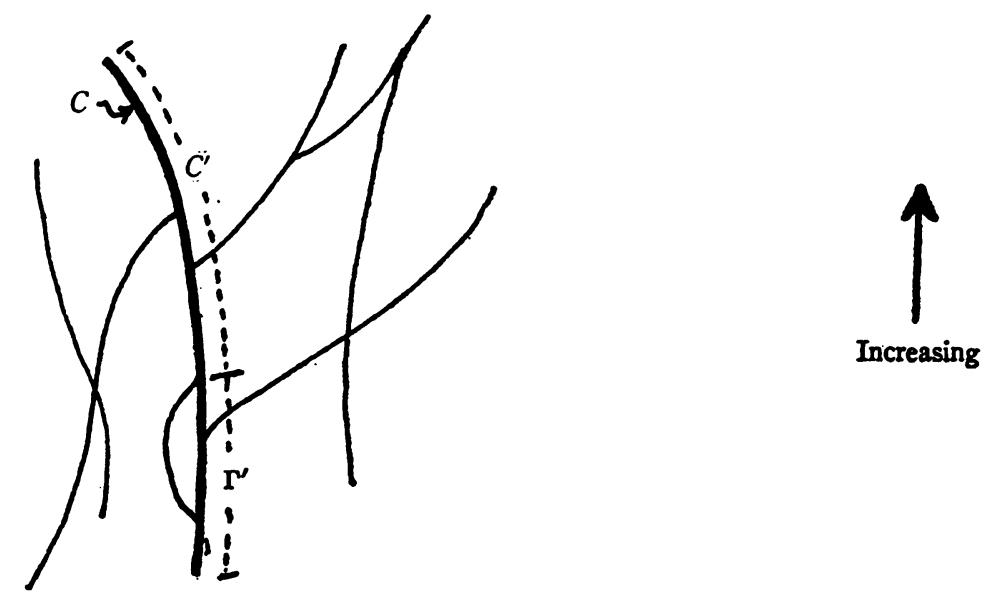

If $\Gamma^{\prime}=\varnothing$, then $C=C^{\prime}$, so for each $\gamma \in C$ there exist elements $\alpha$, $\beta \in \Gamma, \alpha|| \beta$, such that $\alpha<\gamma, \beta<\gamma$. Hence, either there exist elements $\delta_{1}, \delta_{2} \in \Gamma, \delta_{1}|| \delta_{2}$, such that $\delta_{1}<\gamma$ and $\delta_{2}<\gamma$ for all $\gamma \in C$, which implies $C$ is not maximal, or $\Gamma$ has an infinite number of maximal chains. Both cases lead to contradictions so $\Gamma^{\prime} \neq \varnothing$. Moreover, since each $\gamma \in C^{\prime}$ exceeds at least two noncomparable elements of $\Gamma$, and $\Gamma$ has only a finite number of maximal chains we conclude $C^{\prime}$ is not a maximal chain of $\Gamma^{\prime \prime}$. 
Now suppose $D_{i}, 1 \leqq i \leqq m$ are the distinct maximal chains of $\Gamma^{\prime \prime}$. Clearly, no two distinct maximal chains of $\Gamma^{\prime \prime}$ are contained in the same maximal chain of $\Gamma$. Hence, there exist $C_{i}, 1 \leqq i \leqq m$, maximal chains of $\Gamma$ such that $D_{i} \subseteq C_{i}, 1 \leqq i \leqq m$, and $C_{i} \neq C_{j}, i \neq j$. Also, since $C^{\prime}$ is not maximal in $\Gamma^{\prime \prime}$, there is, for each $i$, an element $d_{i} \in D_{i}$ such that $d_{i} \notin C^{\prime}$. But $d_{i} \in \Gamma^{\prime \prime}=\Gamma \backslash \Gamma^{\prime}$ so $d_{i} \notin \Gamma^{\prime}$ and therefore $d_{i} \notin C$, $1 \leqq i \leqq m$. Consequently, $C_{i} \neq C, 1 \leqq i \leqq m$, and $C, C_{1}, \cdots, C_{m}$ are $m+1$ distinct maximal chains of $\Gamma$. Thus, $m+1 \leqq n$ so $m<n$ and $\Gamma^{\prime \prime}$ has fewer than $n$ maximal chains.

Now let $A=V\left(\Gamma^{\prime}, R_{\gamma}\right)$ and $\Delta=V\left(\Gamma^{\prime \prime}, R_{\gamma}\right)$. Then $A$ is an o-group and by induction $\Delta$ is a Riesz group. Moreover, $V\left(\Gamma, R_{\gamma}\right)=A \oplus \Delta$.

For each $v \in \Delta^{+}$, let $\Gamma(v)=\left\{\gamma \in \Gamma^{\prime} \mid \gamma<\delta\right.$ where $v(\delta)$ is a maximal component of $v\}$. Let $H(v)=0$ if $\Gamma(v)=\varnothing$ and $H(v)=\{a \in A \mid S(a)$ $\subseteq \Gamma(v)\}$ if $\Gamma(v) \neq \varnothing$. Then $H(v)$ is a convex $o$-subgroup of $A$. If $u, v \in \Delta^{+}$, then either $\Gamma(u) \subseteq \Gamma(v)$ or $\Gamma(v) \subseteq \Gamma(u)$ since $\Gamma^{\prime}$ is totally ordered. Suppose $u, v \in \Delta^{+}$and $\Gamma(u) \subseteq \Gamma(v)$. Then $H(u) \subseteq H(v)$ and since $u$ and $v$ are positive, $\Gamma(u+v)=\Gamma(v)$ which implies $H(u+v)$ $=H(v)$. Therefore, $H(u)+H(v)=H(v)=H(u+v)$.

For $v \in \Delta^{+}$, let $Q(v)=D I[H(v)]$. Then $Q(v)$ is lower directed since $H(v)$ is an o-group and $Q(u)+Q(v)=D I[H(u)]+D I[H(v)]$ $=D I[H(u)+H(v)]=D I[H(u+v)]=Q(u+v)$ for $u, v \in \Delta^{+}$. Thus, $G=(A, \Delta, f \equiv 0, Q)$ is a Riesz extension.

Finally let $\pi: G \rightarrow V\left(\Gamma, R_{\gamma}\right)$ be defined by $\pi(a, v)=a+v$. Clearly, $\pi$ is an isomorphism of $G$ onto $V\left(\Gamma, R_{\gamma}\right)$. We show both $\pi$ and $\pi^{-1}$ preserve order.

If $(a, v) \in G^{+}$, then $v \geqq \theta$ and $a \in Q(v)$. If the maximal component of $a$ is positive then, $a+v$ is positive in $V\left(\Gamma, R_{\gamma}\right)$, since $v \geqq 0$ in $\Delta$. If the maximal component $a(\delta)$ of $a$ is negative then $0 \geqq a \geqq h \in H(v)$ and by convexity $a \in H(v)$. Thus, there exists $\gamma \in \Gamma$ such that $v(\gamma)$ is a maximal component of $v$ and $\gamma>\delta$. Since $v$ is positive in $\Delta, v(\gamma)>0$, so $a+v$ is positive in $V\left(\Gamma, R_{\gamma}\right)$.

On the other hand, if $0 \leqq \bar{v} \in V\left(\Gamma, R_{\gamma}\right)$, then $\bar{v}=a+v$ where $a \in A$, $v \in \Delta$. Since $\bar{v} \geqq 0$ and no $\gamma \in \Gamma^{\prime}$ exceeds any $\delta \in \Gamma^{\prime \prime}$ it follows that $v \geqq 0$. As before, if the maximal component $a(\delta)$ of $a$ is positive then $a \geqq 0$ and if $a(\delta)$ is negative then, since $a+v \geqq 0$ in $V\left(\Gamma, R_{\gamma}\right), \delta<\gamma$ where $v(\gamma)$ is a maximal component of $v$. Hence, $a \in Q(v)$ and $(a, v)$ $\in G^{+}$. Thus, $\pi$ is an order preserving isomorphism and $V\left(\Gamma, R_{\gamma}\right)$ is a Riesz group. This completes the proof.

The following construction shows if $R_{\gamma}$ is trivially ordered for some $\gamma \in \Gamma$, then additional conditions must be placed on $\Gamma$ so that $V\left(\Gamma, R_{\gamma}\right)$ will be a Riesz group. 
Let $\Gamma=\{\alpha, \beta, \gamma\}$ where $\beta>\alpha, \gamma>\alpha$, and $\beta \| \mid \gamma$. Let $R_{\beta}=R_{\gamma}$ be the naturally ordered reals and $R_{\alpha}$ be the real numbers with the trivial order. Then $V\left(\Gamma, R_{\gamma}\right)$ is not a Riesz group. Now $V=R_{\alpha} \oplus R_{\beta} \oplus R_{\gamma}$ so $v \in V$ can be represented as $v=[v(\alpha), v(\beta), v(\gamma)]$. Clearly, $(0,0,0)$ $<(1,0,1)<(0,0,1)+(0,1,0)$. If $(1,0,1)=\bar{u}+\bar{v}$ where $(0,0,0)$ $\leqq \bar{u} \leqq(0,0,1)$ and $(0,0,0) \leqq \bar{v} \leqq(0,1,0)$ then $\bar{u}(\beta)=\bar{v}(\beta)=0, \bar{v}(\gamma)=0$ and $\bar{u}(\gamma)=1$. Consequently, if $\bar{v} \geqq 0$ then $\bar{v}(\alpha)=0$ and thus $\bar{u}(\alpha)=1$. Hence, $\bar{v}=(0,0,0)$ and $\bar{u}=(1,0,1)$. However, $(1,0,1) \$(0,0,1)$, so $V$ is not a Riesz group.

In $[3$, p. 145] it is shown that if $\Gamma$ is a root system, (for each $\gamma \in \Gamma,\{\delta \in \Gamma \mid \delta \geqq \gamma\}$ is totally ordered), and for $\gamma \in \Gamma, R_{\gamma}$ is a subgroup of the naturally ordered reals, then $V\left(\Gamma, R_{\gamma}\right)$ is an $l$-group. The next example shows $V\left(\Gamma, R_{\gamma}\right)$ is a Riesz group if $\Gamma$ is a root system and $R_{\gamma}$ is a divisible subgroup of the real numbers with either the natural or the trivial order.

We first note that if $G$ is a divisible directed Riesz group, then the Decomposition Property implies the following. If $x, u, v \in G$ and $x<u+v$ then there are elements $\bar{u}, \bar{v} \in G$ such that $x=\bar{u}+\bar{v}$ and $\bar{u}<u, \bar{v}<v$. To show this let $b \in G$ such that $b<0, b<u, b<v, b<x$ and apply the Decomposition Property to $0<x-b-b<(u-b)+(v-b)$. It is also true that if $v>0$ in the above then $\bar{v}$ may be chosen so that $0<\bar{v}<v$.

THEOREM 3.2. If $\Gamma$ is a root system and, for each $\gamma \in \Gamma, R_{\gamma}$ is a divisible subgroup of the real numbers with either the natural or the trivial order, then $V=V\left(\Gamma, R_{\gamma}\right)$ is a Riesz group.

Proof. Suppose $x, u, v \in V^{+}$and $0<x<u+v$. We define $\bar{u}$ and $\bar{v}$ in $V$ so that $x=\bar{u}+\bar{v}$ and $0 \leqq \bar{u} \leqq u, 0 \leqq \bar{v} \leqq v$. Let $w=u+v-x$ and $\Gamma^{\prime}$ be that subset of $\Gamma$ such that $\gamma \in \Gamma^{\prime}$ if and only if $x(\gamma), u(\gamma), v(\gamma)$ and $w(\gamma)$ are maximal components of $x, u, v$ and $w$ respectively. If $\gamma \in \Gamma^{\prime}$, then we have $0<x(\gamma)<u(\gamma)+v(\gamma)$ where $x(\gamma), u(\gamma)$ and $v(\gamma)$ are positive elements of the o-group $R_{\gamma}$. Thus, by the Decomposition Property, there are elements $\bar{u}(\gamma)$ and $\bar{v}(\gamma)$ in $R_{\gamma}$ such that $x(\gamma)$ $=\bar{u}(\gamma)+\bar{v}(\gamma)$ and $0 \leqq \bar{u}(\gamma) \leqq u(\gamma), 0 \leqq \bar{v}(\gamma) \leqq v(\gamma)$.

Let $\Gamma^{\prime \prime}$ be that subset of $\Gamma$ such that $\delta \in \Gamma^{\prime \prime}$ if and only if $w(\delta)$ is a maximal component of $w$ and either $\delta \leqq \lambda<\gamma=\beta$ or $\delta<\lambda=\gamma=\beta$, where $x(\gamma), u(\beta)$ and $v(\lambda)$ are maximal components of $x, u$ and $v$ respectively. For $\delta \in \Gamma^{\prime \prime}$, let $\bar{u}(\delta)$ and $\bar{v}(\delta)$ be defined according to the remarks preceding this theorem, where $\bar{u}(\delta)$ or $\bar{\nu}(\delta)$ is chosen positive when necessary.

Now let $C$ be a maximal chain of $\Gamma$. If the projection of $x$ on $C$ is 
zero let $\bar{u}(\alpha)=\bar{v}(\alpha)=0$ for all $\alpha \in C$. If the projection of $x$ on $C$ is not zero and $x(\gamma), \gamma \in C$ is the maximal component of $x$ on $C$, then $x(\gamma)$ $>0$. Since $x<u+v$, it follows that the projection on $C$ of $u$ or $v$ is not zero and, if $u(\beta), \beta \in C$, is the maximal component of $u$, and $v(\lambda), \lambda \in C$, is the maximal component of $v$, then either $\beta \geqq \gamma$ or $\lambda \geqq \gamma$. In all that follows $x(\gamma), u(\beta), v(\lambda)$ and $w(\delta), \gamma, \beta, \lambda, \delta \in C$, will denote the maximal components of $x, u, v$ and $w$ respectively on $C$.

If $\gamma \in C \cap \Gamma^{\prime}$ let $\bar{u}(\alpha)=\bar{v}(\alpha)=x(\alpha) / 2$ for all $\alpha \in C, \alpha \neq \gamma$. If $\delta \in C \cap \Gamma^{\prime \prime}$ let $\bar{u}(\alpha)=u(\alpha)$ and $\bar{v}(\alpha)=v(\alpha)$ for all $\alpha>\delta$ and $\bar{u}(\alpha)=\bar{v}(\alpha)=x(\alpha) / 2$ for all $\alpha<\delta, \alpha \in C$.

If $u(\alpha)+v(\alpha)=x(\alpha)$ for all $\alpha \in C$, let $\bar{u}(\alpha)=u(\alpha), \bar{v}(\alpha)=v(\alpha)$ for all $\alpha \in C$. If $\beta>\gamma$ and $\lambda>\gamma$, let $\bar{u}(\alpha)=\bar{v}(\alpha)=x(\alpha) / 2$ for all $\alpha \in C$.

If the projection of $v$ on $C$ is zero, or $\beta>\gamma$ and $\lambda \leqq \gamma$, or $\beta=\gamma$, $\lambda<\gamma$ and $\lambda<\delta \leqq \gamma$, let $\bar{u}(\alpha)=x(\alpha)$ and $\bar{v}(\alpha)=0$ for all $\alpha \in C$.

Now clearly, on the chain $C, \bar{u}+\bar{v}=x$ and $0 \leqq \bar{u} \leqq u, 0 \leqq \bar{v} \leqq v$. We perform this construction, or its dual, for each maximal chain of $\Gamma$. A lengthy but straightforward argument shows that $\bar{u}$ and $\bar{v}$ so constructed are well defined elements of $V$ such that $0 \leqq \bar{u} \leqq u$ and $0 \leqq \bar{v} \leqq$.

Thus, $V=V\left(\Gamma, R_{\gamma}\right)$ satisfies the Decomposition Property and $V$ is a Riesz group. This completes the proof.

The following remains an open question: if $\Gamma$ is an arbitrary po-set and for each $\gamma \in \Gamma, R_{\gamma}$ is the real numbers with the natural order, is $V\left(\Gamma, R_{\gamma}\right)$ a Riesz group?

\section{REFERENCES}

1. G. Birkhoff, Lattice-ordered groups, Ann. of Math. 43 (1942), 298-331.

2. - Lattice theory, rev. ed., Amer. Math. Soc. Colloq. Publ. Vol. 25, Amer. Math. Soc., Providence, R. I., 1948.

3. P. Conrad, J. Harvey and C. Holland, The Hahn embedding theorem for abelian lattice ordered groups, Trans. Amer. Math. Soc. 108 (1963), 143-169.

4. L. Fuchs, The extensions of partially ordered groups, Acta. Math. Acad. Sci. Hungar. 1 (1950), 118-124.

5. J. R. Teller, On the extensions of lattice ordered groups, Pacific J. Math. 14 (1964), 709-718.

TUlane University 